\title{
RATIONALIZATION OF SPECTRA OF RARE EARTHS IN CONDENSED MATTER
}

\begin{abstract}
R. REISFELD
Department of Inorganic Chemistry, Hebrew University, 91904 Jerusalem, Israel

AND C.K. J $\not$ RGENSEN

Section of Chemistry, University of Geneva

30 Quai Ansermet, 1211 Geneva 4, Switzerland

The parametrized models are compared with counter-intuitive consequences of the non-relativistic Schrödinger treatment. For both $4 f^{q}, 5 f^{q}$ and $d^{q}$ systems in condensed matter, the energy levels must be strongly influenced by correlation effects. The parametrization of intensities is conceptually very different for $f^{q}$ and $d^{q}$ compounds. The bandwidths of absorption and luminescence are discussed and related to the Born-Oppenheimer factorization.
\end{abstract}

PACS numbers: $71.70 . \mathrm{Ch}$

\section{Introduction}

Around 1955 to 1965 , the opinion prevailed that nearly all transitions in compounds representing oxidation states $[1,2]$ corresponding to a partly filled $3 d, 4 d$ or $5 d$ shell of an element $\mathrm{M}$ having $Z$ at least 22,41 or 73 (and at most 29,47 , or 79 among the coinage metals) are quite broad, but usually not very intense. Exceptions (later rationalized convincingly) occur as a few narrow lines in $3 d^{3}$ chromium(III), manganese(IV), $3 d^{5}$ manganese(II), iron(III), $3 d^{6}$ iron(II), $3 d^{8}$ nickel(II), $4 d^{3}$ molybdenum(III), technetium(IV), $5 d^{3}$ rhenium(IV), iridium(VI), $5 d^{4}$ osmium(IV) and platinum(VI) [3, 4].

The situation was almost opposite in trivalent lanthanides (Ln) from $4 f^{2}$ praseodymium(III) to $4 f^{13}$ ytterbium(III) $(Z=59$ through 70 ) having very sharp absorption bands (which are so weakly dependent on the closest neighbour atoms and on the temperature that they really look like spectral lines of monoatomic species) $[5,6]$. The Slater-Condon-Shortley parameters $[7,8]$ used to describe the numerous $J$-levels in spherical symmetry from $d^{2}$ to $d^{8}$ and from $f^{2}$ to $f^{12}$ (already those four minimum numbers of levels are 9, 9,13 and 13 respectively, to be compared with $J$-levels being 37 of $d^{5}$ and 327 of $f^{7}$ ) are very apt to explain 
the $4 f^{q}$ spectra of differing $\operatorname{Ln}(\mathrm{III})$ [9] but have only indirect [10-12] relevance for $d$ group complexes, even octahedral $\mathrm{MX}_{6}$ in the point group $O_{h}$.

One niveau of interpretation is that $4 f^{q}$ group energy levels are closely similar to $J$-levels in spherical systems, the $(2 J+1)$ mutually orthogonal states (distributed on $(J+1 / 2)$ Kramers doublets, when $q$ is an odd number of electrons) only very slightly separated energy-wise. If we concentrate attention on the $d^{q}$ octahedral $\mathrm{MX}_{6}$, the largest influence on the observed energy levels is exerted by $\Delta$ (the ancient notation [10] was $10 D q$ ), the one-electron energy difference between the two $d$-like orbitals with angular functions $\left(x^{2}-y^{2}\right)$ and $\left(3 z^{2}-r^{2}\right)$ concentrated on the three Cartesian axes; and the less excited three $d$-like orbitals $(x y),(x z)$ and $(y z)$ obviously having the same energy if the names of the Cartesian axes are arbitrarily permuted. The energy difference $\Delta$ usually occurs in the interval 7000 to $40000 \mathrm{~cm}^{-1}(0.9$ to $5 \mathrm{eV}$ ) in a way (called the spectrochemical series) being, to a good approximation $[1,13]$, the product of a central atom parameter increasing smoothly

$$
\begin{gathered}
\mathrm{Mn}(\mathrm{II})<\mathrm{Ni}(\mathrm{II})<\mathrm{V}(\mathrm{II})<\mathrm{Fe}(\mathrm{III})<\mathrm{Cr}(\mathrm{III})<\mathrm{Co}(\mathrm{III})<\mathrm{Mn}(\mathrm{IV}) \\
<\mathrm{Ru}(\mathrm{II})<\mathrm{Mo}(\mathrm{III})<\mathrm{Rh}(\mathrm{III})<\mathrm{Ir}(\mathrm{III})<\mathrm{Re}(\mathrm{IV})<\mathrm{Pt}(\mathrm{IV})
\end{gathered}
$$

and a ligand parameter (only a few examples are given)

$$
\mathrm{Br}^{-}<\mathrm{Cl}^{-}<\mathrm{F}^{-}<\mathrm{OH}^{-}<\mathrm{OH}_{2}<\mathrm{NCS}^{-}<\mathrm{NH}_{3} \ll \mathrm{CN}^{-} \text {. }
$$

These "ligand field" effects almost obliterate the distribution of $d^{q} J$-levels in spherical symmetry. Until the Solvay Meeting in Brussels, May 1956, many chemists tended to believe that $\Delta$ is due to the electrostatic perturbation of the (tiny) non-spherical part of the (huge) Madelung potential due to the charge distribution on surrounding anions. This is almost incompatible with the observation that, if anything, neutral ligands like water, ammonia and amines induce stronger spectrochemical effects than anions do (with the exception of cyanide). For our purpose, the most interesting conclusion is that the lower subshell $(x y),(x z)$, and $(y z)$ and the upper subshell $\left(3 z^{2}-r^{2}\right)$ and $\left(x^{2}-y^{2}\right)$ separated by $\Delta$ in $\mathrm{MX}_{6}$ almost play the role of two different shells, once the symmetry is no longer spherical. Thus, the upper subshell contains (in most $\mathrm{MX}_{6}$ ) to a reasonable approximation $a(=0,1,2,3$ or 4) antibonding electrons, and the lower subshell $q-a(=0,1,2,3,4,5$ or 6$)$ among the $q d$-like electrons.

The Tanabe-Sugano diagrams $[10,14]$ provide, as first pointed out by Orgel [11] a criterium for broad absorption bands, if $a$ increases in the excited state, or, as for $3 d^{5} \mathrm{Mn}$ (II) and $\mathrm{Fe}(\mathrm{III})$, decreases from 2 downwards, in the first excited state. By the same token, broad emission bands occur, when $a$ changes by roughly one unit, and the luminescence "shows a strong Stokes shift. On the other hand, if $a$ remains [15] invariant, rather narrow absorption (and, if detectable, emission) bands correspond to almost parallel curves in the Tanabe-Sugano diagram [14] representing a good imitation of $4 f^{q}$ energy levels, both having minute effects of vibrations.

The "internal" $d^{q}$ transitions are, by no means, the only excitations observed in translucid solutions, glasses and (dilute or stoichiometric) crystals. Some, much more intense, absorption bands have been known for a longer time than the weaker 
$d^{q}$ bands, and are due to electron transfer [16]. The best represented category is due to transfer of one electron (in the sense [17] of configurations differing by the occupation of the orbital in atomic spectroscopy; we do not think about the Mulliken populations in LCAO models of compounds) from the reducing ligand, or collectively from a set of ligands (e.g. six halide ligands) to a partly filled, or empty $d$-shell of a central atom $M$ decreasing its oxidation state [1] by one unit in the excited state. Other types of electron transfer bands [16] are called "inverted", the $\mathrm{M} d^{q}$ losing an electron to a low lying empty molecular orbital of (always) an organic, conjugated ligand. A third type $[16,18,19]$ is due to transfer of an electron from a reducing $\mathrm{M} d^{q}(q=1$ to 10$)$ to another $\mathrm{M}^{*} d^{q}(q=0$ to 9$)$, e.g. from $3 d^{6}$ iron(II) to $3 d^{0}$ titanium(IV) in blue sapphire $\mathrm{Al}_{2-2 \mathrm{x}} \mathrm{Ti}_{\mathrm{x}} \mathrm{O}_{3}$ or black ilmenite $\mathrm{FeTiO}_{3}$, or from $4 d^{10}$ silver(I) to permanganate, $5 d$ group complexes $\mathrm{OsBr}_{6}^{-2}$ or $\mathrm{IrCl}_{6}^{-2}[18]$. It is worth noting that frequent minerals and rocks, which would be white in their iron-free "idealized" formula (as $\mathrm{Na}, \mathrm{Mg}, \mathrm{Al}, \mathrm{K}, \mathrm{Ca}$ silicates) usually are grey to almost black due to simultaneous presence of iron(II) and iron(III) known from greenish black $\mathrm{Fe}(\mathrm{OH})_{2+x}$ and prussian blue $\mathrm{KFe}(\mathrm{CN})_{6} \mathrm{Fe}^{\mathrm{III}}$.

As first observed in coloured, luminescent fluorites $\mathrm{Ca}_{1-x} \operatorname{Ln}_{x} \mathrm{~F}_{2}$ by Przibram [20], lanthanides(II) such as dark green Sm(II), pale yellow Eu(II) and $\mathrm{Yb}$ (II) show strong absorption bands. In analogy to the conditions for Laporte-allowed transitions in atomic spectra, it is agreed today that the excited state belongs to $4 f^{q-1} 5 d$, much like strong absorption bands in the visible of grey uranium(III) acquo-ions, their raspberry-red chloride complexes [21] and U(III) in crystals show the excited configuration $5 f^{2} 6 d$. This type of inter-shell transition is less common in the $3 d$ group, although iron(II) acquo-ions have excited $3 d^{5} 4 s$ states [3], cop$\operatorname{per}(\mathrm{I}) 3 d^{9} 4 s$ (which can be studied [22] by two-photon excitation) and silver(I) $4 d^{9} 5 s$ [23]. A much more intricate question [24] is whether the post-transitional thallium(I), lead(II) and bismuth(III) have $6 s^{2} \rightarrow 6 s 6 p$ excitations to any convincing approximation.

Although lanthanide spectroscopists received strong impulses from the atomic spectroscopists, the occurrence of electron transfer bands of $\operatorname{Ln}($ III) cannot be denied [25]. They are particularly striking in octahedral $\mathrm{LnCl}_{6}^{-3}$ and $\mathrm{LnBr}_{6}^{-3}$ [26] and $\mathrm{LnI}_{6}^{-3}$ [27] studied in acetonitrile solution, and later in cubic elpasolites $\mathrm{Cs}_{2} \mathrm{NaLnX}_{6}$. Electron transfer bands can also be observed [28] in the ultraviolet in europium(III) acquo-ions, sulfate complexes etc., and in crystalline ternary oxides [29] containing europium(III), the relatively most oxidizing $\operatorname{Ln}(\mathrm{III})$. The situation is more clear-cut in $\operatorname{Ln}(\mathrm{IV})$; all yellow to orange cerium(IV) complexes in solution show strong, very broad, absorption bands in the ultraviolet. According to the host lattice, both $4 f^{1}$ praseodymium(IV) and $4 f^{7}$ terbium(IV) can show a range of colours [30] from orange (diluted in cubic $\mathrm{Y}_{2} \mathrm{O}_{3}$ ), chamois (in $\mathrm{Nd}_{2} \mathrm{O}_{3}$ ) to deep purple (in $\mathrm{ThO}_{2}$ ). $\mathrm{Ce}_{0.99} \mathrm{Pr}_{0.01} \mathrm{O}_{2}$ is deep red-brown. Pale violet $\mathrm{Ce}(\mathrm{OH})_{3+x}$ suspended in alkaline solution is likely to be coloured by electron transfer from $\mathrm{Ce}(\mathrm{III})$ to $\mathrm{Ce}(\mathrm{IV})$.

The Ln(III) showing a Laporte-allowed inter-shell transition at the lowest energy is cerium(III). The (unusual) octahedral coordination [26] in $\mathrm{CeCl}_{6}^{-3}$ corresponds to a strong, not exceedingly broad, absorption band at $30300 \mathrm{~cm}^{-1}$ and of $\mathrm{CeBr}_{6}^{-3}$ at $29150 \mathrm{~cm}^{-1}$. The most unexpected result is that the subshell energy 
difference $\Delta$ is at least $15000 \mathrm{~cm}^{-1}$, more than half the observed value $[1,3]$ in pronounced covalent $\mathrm{IrCl}_{6}^{-3}$ and $\mathrm{IrBr}_{6}^{-3}$. The two (only) $J$-levels of $4 f^{1}$ in gaseous $\mathrm{Ce}^{+3}$ occur at 0 and $2253 \mathrm{~cm}^{-1}$ (and the $5 d^{1}$ levels at 49737 and $52226 \mathrm{~cm}^{-1}$ ). Kröger and Bakker pointed out in 1941 that the luminescence of Ce(III) in many glasses and crystals (from the lowest $5 d$-like state) produces a considerable Stokes shift, and two emission maxima separated by about $2000 \mathrm{~cm}^{-1}$ due to $4 f^{1}$. Direct observation of these seven Kramers doublets in absorption is impeded by trace impurities (e.g. producing $\mathrm{OH}$ vibrations).

The five strong absorption bands of the cerium(III) acquo-ion at 39600 , $41700,45100,47400$ and [31] $50000 \mathrm{~cm}^{-1}$ were thoroughly studied by Freed [32] in 1931, and by Heidt and Berestecki [33] and by Okada et al. [34]. The latter authors argue that the excited acquo-ion $\mathrm{Ce}\left(\mathrm{OH}_{2}\right)_{9}^{+3}$ loses a water molecule by a photochemical process during its short mean lifetime (27 nanoseconds) and that this, or a slightly different, $\mathrm{Ce}\left(\mathrm{OH}_{2}\right)_{8}^{+3}$ produces the weak [33] absorption band at $33800 \mathrm{~cm}^{-1}$ (with a concentration [35] of some 4 percent of the cerium). The angular overlap treatment $[6,34,36]$ suggests the "non-bonding" reference energy to be at $29300 \mathrm{~cm}^{-1}$, and that the main ennea-acquo-ion has the structure known [32] from $\mathrm{La}\left(\mathrm{OH}_{2}\right)_{9}\left(\mathrm{C}_{2} \mathrm{H}_{5} \mathrm{OSO}_{3}\right)_{3}$.

Recently, the 11-coordinated tysonite-type $\mathrm{CeF}_{3}$, as well as $\mathrm{La}_{1-x} \mathrm{Ce}_{x} \mathrm{~F}_{3}$ attracts much attention [37, 38]. The highly diluted crystal $(x=0.0005$ at $10 \mathrm{~K})$ emits at $32000 \mathrm{~cm}^{-1}$ and shows five absorption bands (each due to one Kramers doublet) at $40500,43100,45900,48300$, and $52400 \mathrm{~cm}^{-1}$. The four first values are 800 to $1400 \mathrm{~cm}^{-1}$ above the ennea-acquo-ion. One has to be careful to avoid defects and traces of oxide in $\mathrm{CeF}_{3}$. Low concentrations of cerium(III) in glasses also are luminescent strongly in the ultraviolet [5] and transfer energy as for instance to terbium(III) and to thulium(III) in borate glasses.

\section{Good and bad news about the non-relativistic Schrödinger treatment}

Atomic spectroscopists (and astrophysicists) are accustomed to gas pressure, the concentration of the atom emitting the spectral line, and stray electric fields usually being sufficiently weak that extrapolation to the practically unperturbed atom is feasible. Condensed matter, even with one luminescent molecule or polyatomic ion being confined on a given site, e.g. in sol-gel glasses [39] might still play some tricks.

From the Schrödinger perspective [40] a monoatomic entity consists of one nucleus with $Z$ times the protonic charge, and $K$ electrons, and hence an external charge $z=Z-K$. When $K$ is above 1 , one can expect quite serious effects of configuration interaction and correlation effects $[41,42]$. When a chemist speaks about the electron configuration, she or he means the preponderant configuration [1] correctly predicting the low lying $J$-levels (and at least to a fair approximation, their relative order) including the ground state.

A chemical system contains for the Schrödinger series of nuclei with (identical or differing) $Z_{1}, Z_{2}, Z_{3}, \ldots, Z_{N}$ and $K^{*}$ electrons. In a neutral molecule, $K^{*}$ equals the sum of all the various values of $Z$. A system with many thousands 
of nuclei has to be almost electrically neutral, but for a small number of nuclei, $K^{*}$ can legitimately be a few units lower or higher than the $Z$ sum. We assume now the Born-Oppenheimer approximation (based on the fortunate fact that each nucleus is at least 1800 times heavier than an electron); for a monoatomic entity, the Schrödinger wave function then is the product of a translational factor (function of three Cartesian coordinates) and an electronic factor (not allowing the slightest discernibility of electrons as individuals) and going in a space with $3 K$ variables ( 240 for a mercury atom), besides the two-valued spin-variable $m_{s}$ for each electron [7].

With more than one nucleus, there is also a rotational factor and a vibrational factor, if we accept the Born-Oppenheimer model. For two nuclei, the rotational factor has two degrees of freedom, and the vibrational factor (one internuclear distance) one. (We consider here nuclei as Euclidean points.) With $N$ nuclei (at least 3) there are $3 N-6$ mutually independent internuclear distances, and the vibrational factor has this number of degrees of freedom, and rotation has three. The $K^{*}$ electrons have an electronic function with $3 K^{*}$ dimensions needed, an ethanol molecule 78 dimensions.

As children, we got spoiled with diatomic molecules. Their potential curve has one variable, the internuclear distance. This has pervaded books on band shapes and luminescence, discussing "breathing motion" along one totally symmetric vibrational motion. However, no imperial decree can remove the fact that $\mathrm{MX}_{4}$ (and other systems with 5 nuclei) have a potential surface (or, if you prefer, hypersurface): the inergy as function of 9 independent internuclear distances, hence subsisting in a 10-dimensional space; neither that $\mathrm{MX}_{6}$ with 7 nuclei has a potential surface subsisting in 16 dimensions, nor that $\mathrm{CH}_{3} \mathrm{CH}_{2} \mathrm{OH}$ and its isomer $\mathrm{CH}_{3} \mathrm{OCH}_{3}$ are the Schrödinger solutions to the same 22-dimensional potential hypersurface. This makes the organic idea "path of reaction" rather metaphysical; we can only recognize a path of reaction after having compared with all the alternatives.

From a strictly deductive point of view [40] the potential surface should have the Schrödinger eigenenergy evaluated in each point. This means, in practice, a fine grid of points, and emphasis on behaviour close to the minima of the surface in the $(3 N-5)$-dimensional space. This approach may have some appeal in horribly complicated cases of $\mathrm{K}^{*}=2$ such as $\mathrm{H}_{3}^{+}$or $\mathrm{H}_{2} \mathrm{He}^{+2}$. But we have to recognize today that our choice of favourite models hardly is based on good approximations to the time-independent non-relativistic Schrödinger equation, but on inductive treatment (supported to some extent by comparison with spectroscopic experience). It should be added that negative conclusions in a way can be constructive [43]; for instance, the absorption band shape (distribution of oscillator strength [44]) is a Franck-Condon projection up on an excited potential surface; the luminescence is a downward projection from a (more or less) thermalized vibronic dispersion near its minimum down to lower potential surfaces with entirely different slopes, providing large Stokes shifts [14] and unexpected shape of the emission band. The "excited state absorption" goes from the same thermalized minimum [44] up to higher potential surfaces (having one or several minima), and photochemical reac- 
tions correspond to asymptotic behaviour of a set of internuclear distances entirely different from the original ground state.

\section{Which inductive models are attractive and informative ?}

Even without entering the moot question whether biology can be reduced to chemistry, the more polite question whether chemistry can be reduced to quantum mechanics has been muddled up to a great extent by the recent idolatry of huge supercomputers. Including correlation effects [40-42] we are far from moderately precise wave functions for one nucleus and a number of electrons significantly above $K=30$. This limit is not dramatically worse for several nuclei and the same $K^{*}$. Unfortunately, for chemists (and their relations with Schrödinger), the heat of atomization per atom $H_{\mathrm{a}}$ of a gaseous molecule or a solid is never above $9 \mathrm{eV}$, less than 0.001 times the binding energy of the 15 electrons in a phosphorus atom, and less than $10^{-4}$ of 39 electrons in yttrium. We are aware $[45,46]$ that some procedures of quantum chemistry (in many of the attempts for at most 5 nuclei, among which at most one has $Z$ above 10 , and $K^{*}$ below 25) agree better than $0.2 \mathrm{eV}$ for total atomization energies. However, there is a feature of Russian roulette that the results are not guaranteed by the variational principle. Typically, chemical problems like stereochemistry or relative stability of several isomers are far more difficult.

Looking back on $4 f^{q}$ and $d^{q}$ spectroscopy growing up since 1954, the two areas have surprisingly different "standard models". The $(2 J+1)$ states of each $J$-level remain atomic spectroscopic (gaseous $\mathrm{Pr}^{+3}$ is the only $\mathrm{Ln}^{+3}$ with all 13 levels determined; $\mathrm{Pr}^{+2}$ isoelectronic with $\mathrm{Nd}^{+3}$ has 38 identified levels [47] out of 41). The Slater-Condon-Shortley parameters are the same (within 3 percent) in $\operatorname{Ln}(\mathrm{III})$ and $\mathrm{Ln}^{+3}$. Technically, the demise of the model [13] of the minute non-spherical part of the Madelung potential as source of weak energy differences in $\operatorname{Ln}(\mathrm{III})$ and $\operatorname{Ln}(\mathrm{II})$ compounds has been remedied $[48,49]$ by the same angular overlap model as $d^{q}$, but the antibonding effects 50 times smaller, and not decided mainly by the kind of ligand $\mathrm{X}$, but rather by a strong decrease for longer $\mathrm{Ln}-\mathrm{X}$ distances. It may be added that transthorium elements $\mathrm{M}$ (III) are quite similar to $J$-levels of $\mathrm{Ln}(\mathrm{III})$, but higher oxidation states $\mathrm{U}(\mathrm{IV}), \mathrm{U}(\mathrm{V}), \mathrm{Np}(\mathrm{IV}), \mathrm{Np}(\mathrm{V})$, $\mathrm{Np}(\mathrm{VI}), \mathrm{Pu}(\mathrm{IV})$... have stronger antibonding effects on $5 f^{q}$ than $\mathrm{M}(\mathrm{III})$, but again determined by shorter $\mathrm{M}-\mathrm{X}$ distances, e.g. in $\mathrm{UCl}_{6}^{-2}$.

Transition probabilities and oscillator strengths of "internal" $4 f^{q}$ and $d^{q}$ absorption bands show an entirely different origin. The parameters $[5,6]$ of a model proposed independently by Judd and by Ofelt in 1962 are three $U_{t}$ evaluated from the $J$-level positions and the pure $4 f^{q}$ wave function (disregarding correlation effects) and three material $\Omega_{t}$ from the overdetermined expression $\left(\Omega_{2} U_{2}+\Omega_{4} U_{4}+\right.$ $\Omega_{6} U_{6}$ ) when the oscillator strengths of four or more excited $J$-levels are studied. There is no doubt [6] that the $\Omega_{t}$ are influenced by weak covalent bonding, and $\Omega_{2}$ (being enormous in the case of "pseudoquadrupolar hypersensitive transitions") makes the electric quadrupolar transition [7] far more probable than in vacuum. With exception of a few low-symmetry $d^{q}$ compounds [3] the major mechanism involves a second-order perturbation with the positions of the electron transfer 
$(X \rightarrow M)$ bands $[16,50]$, the transmitted intensity inversely proportional to the square of the wave-number difference, and (by accident, or for an intrinsic reason) the non diagonal element being similar to the width of the $d^{q}$ absorption band.

$4 f^{q}$ electron transfer bands $[5,16,25-29]$ tend to be 10 or 20 times weaker in $\operatorname{Ln}(\mathrm{III})$ than in $d^{q}$ complexes. This difference is not paradoxical in view of the large ratio between the $\mathrm{Ln}-\mathrm{X}$ distance and the average $4 f$ radius, but $\mathrm{Ce}(\mathrm{IV}), \mathrm{Np}$ (IV) and $\mathrm{Pu}(\mathrm{IV})$ hexahalides [51] have electron transfer bands almost as strong as analogous $d^{q}$ cases. There is no striking intensification of $4 f^{q}[25]$ or $5 f^{q}$ "internal" transitions in the vicinity of the electron transfer bands.

Obviously, inter-shell transitions in $\operatorname{Ln}(\mathrm{II})$ and $\operatorname{Ln}(\mathrm{III}) 4 f^{q} \rightarrow 4 f^{q-1} 5 d$ [25-27] and in U(III) [21] and M(IV) $5 f^{q} \rightarrow \Phi 5 f^{q-1} 6 d$ transitions [51] involve mixtures of characteristics of "internal" transitions in partly filled $4 f$ and $3 d, 4 d$ or $5 d$ shells. Thus, the one-electron energy difference between the five $5 d$ - or $6 d$-like orbitals provide moderately broad bands [5, 21, 26, 27, 31-35] presumably having a mechanism rather similar to the change of the number $a$ of antibonding electrons in the Tanabe-Sugano diagram $[10,14 ; 43,44]$. It should not be accepted (without first pondering a moment) that the bandwidth of absorption (and of any luminescence) is due exclusively to "breathing" multiplying all the ground state $\mathbf{M}-\mathrm{X}$ distances (in a given instant) by the same factor close to 1. Like 16-dimensional potential surfaces of $d^{q}$ states and of electron transfer states may cross each other at surrealistic combinations of the 15 internuclear distances in $\mathrm{MX}_{6}$, it may also be the case that $4 f^{q}$ and $4 f^{q-1} 5 d$ (not to speak about $4 f^{q-2} 5 d^{2}$ ) may cross each other in lanthanides.

Although the "ligand field" effects [48, 49] separating individual sublevels of a given $J$-level are minute, when conditioned by the Frank-Condon projection from the ground $J$-level to higher lying $J$-levels, it cannot be excluded that the geometry of some of the sublevels may be highly distorted, providing broad, asymmetric or multi-peak emission bands. This is related to the complicated problem of unusually pronounced co-excited vibrations [35-52] especially in gadolinium(III) luminescence, also when provoked by $\mathrm{X}$-rays. The excitation spectrum for emission of praseodymium(III) from ${ }^{3} P_{0}$ to ${ }^{3} F_{2}$ and to ${ }^{3} H_{6}$ show unusual vibronic structure [53].

Seen from a generalist perspective, such phenomena are side-effects of the imperfect separation of vibronic and electronic factors in the Born-Oppenheimer model.

\section{Acknowledgment}

We are grateful to the Swiss National Science Foundation for their grant 20.32127.91 rendering the collaboration between the universities in Geneva and Jerusalem feasible.

\section{References}

[1] C.K. Jфrgensen, Oxidation Numbers and Oxidation States, Springer, Berlin 1969.

[2] C.K. J申rgensen, Z. Anorg. Allg. Chem. 540, 91 (1986). 
[3] C.K. Jфrgensen, Adv. Chem. Phys. 5, 33 (1963).

[4] G.C. Allen, K.D. Warren, Struct. Bonding 9, 49 (1971); 19, 105 (1974).

[5] R. Reisfeld, C.K. Jфrgensen, Lasers and Excited States of Rare Earths, Springer, Berlin 1977.

[6] R. Reisfeld, C.K. Jфrgensen, in: Handbook on the Physics and Chemistry of Rare Earths, Eds. K.A. Gschneidner, L. Eyring, Vol. 9, North-Holland, Amsterdam 1987, p. 1.

[7] E.U. Condon, G.H. Shortley, Theory of Atomic Spectra, 2nd ed., Cambridge University Press, Cambridge 1953.

[8] M. Brorson, C.E. Schäffer, Inorg. Chem. 27, 2522 (1988).

[9] W.T. Carnall, P.R. Fields, K. Rajnak, J. Chem. Phys. 49, 4412, 4424, 4443, 4447, 4450 (1968).

[10] Y. Tanabe, S. Sugano, J. Phys. Soc. Jpn. 9, 753, 766 (1954).

[11] L.E. Orgel, J. Chem. Phys. 23, 1004, 1819, 1824, 1958 (1955).

[12] J.S. Griffith, Theory of Transition-Metal Ions, Cambridge University Press, Cambridge 1961.

[13] C.K. J $\phi$ rgensen, Modern Aspects of Ligand Field Theory, North-Holland, Amsterdam 1971.

[14] R. Reisfeld, C.K. Jфrgénsen, Struct. Bonding 69, 63 (1988).

[15] R. Reisfeld, A. Kisilev, C.K. Jorgensen, Chem. Phys. Lett. 111, 19 (1984).

[16] C.K. Jфrgensen, Prog. Inorg. Chem. 12, 101 (1970).

[17] C.K. Jorgensen, Struc. Bonding 22, 49 (1975).

[18] C.K. Jorgensen, Acta Chem. Scand. 17, 1034 (1963).

[19] G. Blasse, Struct. Bonding 76, 153 (1991).

[20] K. Przibram, Z. Phys. 102, 331 (1936); 107, 709 (1937).

[21] C.K. Jфrgensen, Acta Chem. Scand. 10, 1503 (1956).

[22] D.S. McClure, Acta Phys. Pol. A 71, 787 (1987).

[23] J. Texter, J.J. Hastreiter, J.I. Hall, J. Phys. Chem. 87, 4690 (1983).

[24] G. Boulon, C.K. Jorgensen, R. Reisfeld, Chem. Phys. Lett. 75, 24 (1980).

[25] C.K. Jorgensen, Mol. Phys. 5, 271 (1962).

[26] J.L. Ryan, C.K. Jфrgensen, J. Phys. Chem. 70, 2845 (1966).

[27] J.L. Ryan, Inorg. Chem. 8, 2053 (1969).

[28] J.C. Barnes, P. Day, J. Chem. Soc., 3886 (1964).

[29] G. Blasse, Struct. Bonding 26, 43 (1976).

[30] H.E. Hoefdraad, J. Inorg. Nucl. Chem. 37, 1917 (1975).

[31] C.K. Jфrgensen, J.S. Brinen, Mol. Phys. 6, 629 (1963).

[32] S. Freed, Phys. Rev. 38, 2122 (1931).

[33] L.J. Heidt, J. Berestecki, J. Am. Chem. Soc. 77, 2049 (1955).

[34] K. Okada, Y. Kaizu, H. Kobayashi, K. Tanaka, F. Marumo, Mol. Phys. 54, 1293 (1985).

[35] C.K. J $\phi$ rgensen, in: Handbook on the Physics and Chemistry of Rare Earths, Eds. K.A. Gschneidner, L. Eyring, Vol. 11, North-Holland, Amsterdam 1988, p. 197. 
[36] C.E. Schäffer, Pure Appl. Chem. 24, 361 (1970).

[37] C. Pedrini, B. Moine, J.C. Gacon, B. Jacquier, J. Phys. Condens. Matter 4, 5461 (1992).

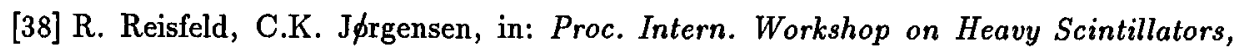
"Crystal 2000", Chamonix 1992, Editions Frontières, Gif-sur-Yvette 1993, p. 155.

[39] R. Reisfeld, C.K. Jфrgensen, Struct. Bonding 77, 207 (1992).

[40] C.K. Jorgensen, Comments Inorg. Chem 12, 139 (1991).

[41] C.K. J $\phi$ rgensen, Chimia 42, 21 (1988).

[42] C.K. Jorgensen, Chimia 46, 444 (1992).

[43] C.K. Jorgensen, J. Phys. (Paris) Coll. C7, 443 (1991).

[44] C.K. Jфrgensen, Opt. Soc. Am. Proc. Tunable Solid State Lasers (Washington DC) 5, 252 (1989).

[45] L.A. Curtiss, C. Jones, G.W. Trucks, K. Raghavachari, J. Pople, J. Chem. Phys. 93, 2537 (1990).

[46] W. Klopper, W. Kutzelnigg, J. Chem. Phys. 94, 2020 (1991).

[47] R.E. Trees, J. Opt. Soc. Am. 54, 651 (1964).

[48] C.K. Jфrgensen, R. Pappalardo, H.H. Schmidtke, J. Chem. Phys. 39, 1422 (1963).

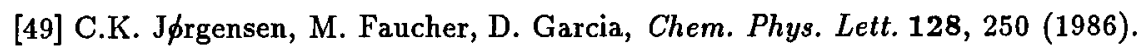

[50] R.F. Fenske, J. Am. Chem. Soc. 89, 252 (1967).

[51] J.L. Ryan, C.K. Jфrgensen, Mol. Phys. 7, 17 (1963).

[52] G. Blasse, L.H. Brixner, G. Hyatt, Chem. Phys. Lett. 164, 617 (1989).

[53] C. de Mello Donega, G. Blasse, Chem. Phys. Lett. 183, 367 (1991). 